\title{
Prospects for using flour from amaranth seeds in dessert technology
}

\author{
V.S. Kutsenkova ${ }^{1, *}$, N.M. Kuprik ${ }^{2}$, and N.V. Nepovinnykh ${ }^{1}$ \\ Saratov State Agrarian University named after N.I. Vavilov, 410012, 1, Theatre sq., Saratov, \\ Russian Federation
}

\begin{abstract}
Amaranth is a pseudo-grain with a high grain yield that can withstand extreme climatic and soil conditions. Amaranth also has good nutritional qualities with high levels of protein, minerals and fat compared to commonly used grains such as corn. Amaranth grain contains $16 / 100 \mathrm{~g}$ protein, $173 \mathrm{mg} / 100 \mathrm{~g}$ calcium, $35 \mathrm{mg} / 100 \mathrm{~g}$ iron, $3 \mathrm{mg} / 100 \mathrm{~g}$ zinc and higher levels of potassium, phosphorus, magnesium, manganese, vitamins $\mathrm{A}$ and $\mathrm{E}$ and folic acid than grain of cereals. Consuming this grain helps in improving kidney complaints, constipation and anemia. The aim of this study is to develop a dessert technology with the addition of a plant additive amaranth seeds sort "Polet" as a complete protein and a source of dietary fiber and replacing sugar with flower honey. Optimum concentration of flour from amaranth seeds was determined. During the experiment, the textural properties of desserts were studied.
\end{abstract}

\section{Introduction}

Human health is directly related to the food that he eats on a daily basis. For his normal life, proper nutrition is of great importance, creating the necessary conditions for optimal well-being, maintaining good health and working capacity of the human body.

The growing industrialization and scientific and technological progress of the civilization of mankind from the point of view of medicine are no longer evaluated as an exclusively progressive phenomenon. Along with the unfavorable impact of technogenic and anthropogenic factors, insufficient human consumption of natural food components comes to the fore, which leads to metabolic disorders and numerous diseases. With this quality of nutrition, the population of developed countries, including Russia, begins to degrade both in terms of a natural decline in numbers and deterioration in the gene pool [13].

From the concept of healthy nutrition of the population of the Saratov region, it was found that the contribution of unbalanced nutrition to mortality is $12.9 \%$, and overweight $12.5 \%, 55 \%$ of the adult population are overweight, which is 2 times higher than the average Russian indicator (Saratov region - 15.4 per thousand of the population, the Russian Federation - 7.9 per thousand of the population).

\footnotetext{
*Corresponding author: author@email.org
} 
One of the ways to improve the quality of nutrition is to introduce food additives with high taste and nutritional benefits into the diet, which include processed products of amaranth seeds.

Amaranth culture has long been recognized as a promising genus of plants that can provide the human body with high-quality protein, polyunsaturated fatty acids, squalene and other valuable components. Amaranth is known not only for its environmental sustainability, but also for its excellent nutritional properties, thanks to which it attracts the increased attention of technologists. Currently, more than 100 species of amaranth are known.

Amaranth is a pseudo-grain with a high grain yield that can withstand extreme climatic and soil conditions. Amaranth also has good nutritional qualities with high levels of protein, minerals and fat compared to commonly used grains such as corn. Amaranth grain contains 16/100 g protein, $173 \mathrm{mg} / 100 \mathrm{~g}$ calcium, $35 \mathrm{mg} / 100 \mathrm{~g}$ iron, $3 \mathrm{mg} / 100 \mathrm{~g}$ zinc and higher levels of potassium, phosphorus, magnesium, manganese, vitamins $\mathrm{A}$ and $\mathrm{E}$ and folic acid than grain of cereals. Amaranth proteins are composed mainly of three main fractions (albumins, globulins and glutelins) with little or no prolamine.

Amaranth grain is the most important component of globulins, accounting for $90 \%$ and approximately $19 \%$ of the total amount of grain proteins. Although amaranth grain is a high glycemic ingredient, it is a rich source of unsaturated fatty acids and polyphenols (flavonoids) that have cholesterol-lowering and antioxidant activity. Consuming this grain helps in improving kidney complaints, constipation and anemia [4-9].

New amaranth seeds sort "Polet" of the Saratov selection used in this study is the development of scientists of the Russian Research and Design Technological Institute of Sorghum and Maize, Saratov, the new sort is included in the State Register of Russia.

According to WHO, about 1 billion people on the planet suffer from overweight and 300 million of them suffer from obesity. More than 200 million people suffer from diabetes, which is a consequence of excessive consumption of easily digestible carbohydrates, among which in the first place is sucrose. Technologists and scientists have made numerous attempts to reduce or replace sugar in food products with various sweeteners. Flower honey is a natural substance produced by bees, mainly Apis mellifera L., and is a nutritious product of economic importance throughout the world. The main components of honey are sugars, mainly fructose and glucose, although other minor components such as enzymes, proteins, organic acids, minerals, pollen grains, waxes and phytochemicals are also present. Some of these components are naturally found in nectar (or pollen), while others are introduced by bees during the honey maturation process, thereby determining differences in the composition and functional properties of honey. Many studies have shown that honey has an antibacterial effect due to its high osmolarity, low $\mathrm{pH}$, hydrogen peroxide content and the content of other, uncharacteristic compounds for it. The low water activity of honey inhibits the growth of most bacteria, but this is not the only explanation for its antimicrobial activity. Honey has anti-inflammatory, tonic, antiinflammatory, expectorant, analgesic and tonic properties, improves the immune system, disinfects, heals wounds and burns. It is also used to treat diseases of the cardiovascular system, kidneys, liver, biliary tract, and intestines.

The development of dessert technology with the addition of amaranth seed flour will expand the range of functional products. As a prototype product, a classic dessert was chosen, produced according to traditional technology, including the use of the following ingredients in the formulation: sour cream, milk, eggs, sugar, gelatin, vanillin.

The aim of this study is to develop a dessert technology with the addition of a plant additive as a complete protein and a source of dietary fiber - flour from amaranth seeds sort «Polet» and replacing sugar with flower honey as a vitamin supplement. 


\section{Methods}

Amaranth seeds sort "Polet", flower honey and dessert (control and experimental samples) were used us materials. Amaranth flour was obtained from amaranth seeds sort «Polet» using a technology which includes the following stages: washing, drying, cooling and grinding.

Safety indicators (GMO) of flour from amaranth seeds sort "Polet" were determined by the PCR method. Test systems were used to qualitatively and quantitatively determine the content of genetically modified organisms in food and food products. The method is aimed at identifying regular sequences (35S promoter of cauliflower mosaic virus), DNA sequences characteristic of soybeans, as well as determining the ratio of $35 \mathrm{~S}$ promoter and soybean DNA. The method for detecting GMOs of plant origin in food products and food raw materials of plant origin using test systems is based on the use of polymerase chain reaction with the detection of amplification results after completion of the reaction using a PCR detector or in "real time" using a detecting amplifier.

The amino acid composition of protein of amaranth seed was analyzed by HPLC on a Dionex Ultimate 3000 Thermo Scientific chromatograph (USA) using a Nucleodur HTec C18 reverse-phase column, the average particle diameter was $5 \mu \mathrm{m}$ with $100 \AA$ pores, a 150 * $3.0 \mathrm{~mm}$ geometry (Macherey-Nagel). Amino acids were identified using a precolumn dansyl chloride modification. This method provides for amino acid derivatization to form stable derivatives, intensively absorbing light between 310 and $350 \mathrm{~nm}$, and is characterized by high sensitivity and high separation efficiency. The biological value of proteins was evaluated by a chemical method of calculating the amino acid score, based on a comparison of the amino acid composition of the studied protein with the reference amino acid scale of the ideal protein established by FAO/WHO.

The texture properties of the finished desserts were studied using a texture analyzer (Stable Micro System, TA.XTplus, England) equipped with a $5 \mathrm{~kg}$ strain gauge. The reverse immersion test was carried out using a $35 \mathrm{~mm}$ diameter probe (sample code: A / BE-d35) and a sample size of $4 \times 2 \times 2 \mathrm{~cm}$. The samples were subjected to two successive cycles at a pressure equivalent to $50 \%$ of the sample height. The time between the two cycles was $15 \mathrm{~s}$ and the test speed was $1 \mathrm{~mm} / \mathrm{s}$. The quantitative parameters extracted from the force-time curve were: strength (maximum peak force in the first cycle), cohesion (peak negative force in the first cycle), viscosity index (negative area in the first cycle), viscosity (area to a positive peak in the first cycle).

In this study, sugar was completely replaced by flower honey.

Concentration of sweetener was determined according equation:

$$
\mathrm{C}_{\mathrm{sw}}=\mathrm{C}_{\mathrm{sucrose}} / \mathrm{D}_{\mathrm{sw}},
$$

where $\mathrm{C}_{\mathrm{sw}}-$ concentration of sweetener, $\%$

$\mathrm{C}_{\text {sucrose }}$ - amount of sucrose in the product according recipe, $\%$

$\mathrm{D}_{\mathrm{sw}}$ - degree of sweetness

Calorie content was determined using chemical composition according equation:

$$
\text { Calorie content }=9 * \mathrm{X} 1+4 * \mathrm{X} 2+4 * \mathrm{X} 3+2 * \mathrm{X} 4,
$$

where X1 - mass fraction of fat in the product, $\mathrm{g} / 100 \mathrm{~g} ; \mathrm{X} 2$ - mass fraction of protein in the product, $\mathrm{g} / 100 \mathrm{~g} ; \mathrm{X} 3$ - mass fraction of mono and disaccharides in the product, $\mathrm{g} / 100 \mathrm{~g}$; $\mathrm{X} 4$ - mass fraction of dietary fibre in the product, $\mathrm{g} / 100 \mathrm{~g}$. 
Water activity (aw) of desserts was measured with water activity meter (Novasina, Lab Master, Swiss).

Moisture content (W) of desserts was determined using a moisture analyzer "Evlas 2M" (Russia). Three grams of sample was loaded on an aluminium plate and heated at 160 ${ }^{\circ} \mathrm{C}$ for 5 min.

Reliability of results obtained is ensured by the use of modern instruments and methods of analysis, approbation of technologies under production conditions, repeatability, and mathematical processing of experimental data using an SPSS system. Results were considered significant if $\mathrm{p}<0.05[10]$.

\section{Results and discussion}

In addition to amaranth, other raw materials of the Saratov region were also studied. The results of the study are shown in Figure 1. Safflower, buckwheat, flax, peas and amaranth were tested for GMO content.

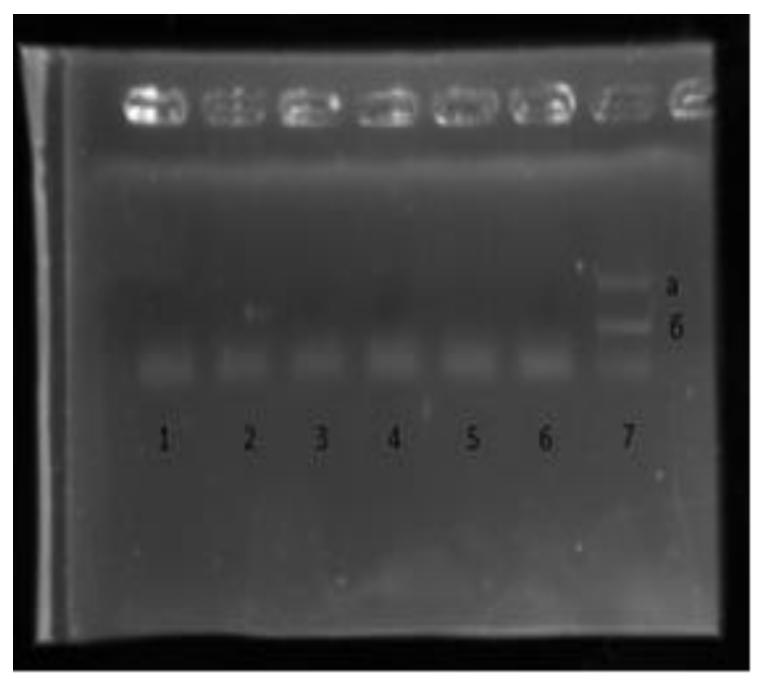

Fig.1. The result of the analysis of regional plant raw materials for the content of GMOs; a - internal control (soy DNA); b - GMO marker (35S promoter); 1 - negative control sample; 2 - safflower seeds; 3 - buckwheat; 1 - flax seeds; 5 - peas; 6 - amaranth seeds sort "Polet"; 7 - positive control sample.

Figure 1 shows that amaranth seeds sort «Polet» (position 6) does not contain GMOs in its composition.

Nutritional value and calorie content per $100 \mathrm{~g}$ of amaranth seeds sort "Polet" was studied, data is presented in Table 1.

Table 1. Nutritional value and calorie content per $100 \mathrm{~g}$ of amaranth seeds sort "Polet".

\begin{tabular}{|c|c|}
\hline Nutrients & Contents \\
\hline Proteins, $g$ & 11,26 \\
\hline Fat, $g$ & 7 \\
\hline Carbohydrates, $g$ & 58,6 \\
\hline Calorie content, kcal & 371 \\
\hline
\end{tabular}


The amino acid composition of proteins of amaranth seeds sort "Polet" was studied. Results showed that this sort contains in its composition an almost complete set of essential amino acids, presented in Table 2.

Table 2. Amino acid composition of proteins of amaranth seeds sort "Polet".

\begin{tabular}{|c|c|c|c|}
\hline Amino acid & $\begin{array}{c}\text { Molecular weight, g } \\
\text { / mol }\end{array}$ & $\begin{array}{c}\text { Mass fraction of } \\
\text { amino acid in the } \\
\text { sample, g / 100 g } \\
\text { of product }\end{array}$ & $\begin{array}{c}\text { Daily } \\
\text { requirement, } \%\end{array}$ \\
\hline Aspartic acid & 133,1 & 1,24 & 20,67 \\
\hline Serine & 105,1 & 0,73 & 24,33 \\
\hline Glutamic acid & 147,1 & 1,97 & 39,40 \\
\hline Glycine & 75,07 & 0,68 & 22,67 \\
\hline Histidine & 155,2 & 0,27 & 13,50 \\
\hline Threonine & 119,1 & 0,49 & 16,33 \\
\hline Arginine & 174,2 & 1,27 & 21,17 \\
\hline Alanin & 89,06 & 0,43 & 14,33 \\
\hline Proline & 115,1 & 0,52 & 10,40 \\
\hline Cysteine & 120,15 & 0,21 & 7,00 \\
\hline Tyrosine & 181,2 & 0,29 & 7,25 \\
\hline Valine & 117,1 & 0,58 & 14,50 \\
\hline Methionine & 149,2 & 0,25 & 8,33 \\
\hline Lysine & 146,2 & 0,62 & 13,50 \\
\hline Isoleucine & 131,2 & 0,53 & 296,09 \\
\hline Leucine & 131,2 & 0,67 & 11,26 \\
\hline Phenylalanine & 165,2 & & 0.40 \\
\hline & & & 0 \\
\hline
\end{tabular}

As can be seen from Table 2, of amaranth seeds sort "Polet", in their composition contain an almost complete set of essential amino acids, except for tryptophan, since this amino acid is found in dairy products. High content of hydrophobic amino acids in amaranth seeds (valine, leucine, isoleucine, phenylalanine, methionine) causes their tendency to form with fatty acids lipid-protein complexes, representing are complex associates. It can be assumed that lipid-protein complexes will be components that contribute to better structure formation of dessert due to hydrophobic interactions. On the other hand, the polar side groups of amino acid residues (lysine, threonine, cysteine, tyrosine) will also provide the binding of protein macromolecules to each other due to electrostatic interaction.

Sensory properties of amaranth seeds sort "Polet" were investigated, data are presented in Table 3. 
Table 3. Sensory properties of amaranth seeds sort "Polet".

\begin{tabular}{|l|l|}
\hline \multicolumn{1}{|c|}{ Indicator } & \multicolumn{1}{c|}{ Characteristic of sensory properties } \\
\hline Appearance & Crushed bulk product without foreign and large inclusions \\
\hline Color & Creamy white \\
\hline Smell & Peculiar to the smell of amaranth, without foreign smell \\
\hline
\end{tabular}

In the course of the experiment, formulations and technology for the preparation of dessert with various concentrations of amaranth seeds sort "Polet" (2, 5, 7 and 10\%) and flower honey $(10,13$ and $17 \%)$ were developed. In the course of the experiment, the optimal concentrations of honey $-13 \%$ and a food additive from amaranth seeds - $10 \%$ were established; at these concentrations, the dessert retains its texture well and has a moderately sweet taste.

Texture plays an important role in the creation of structured products. The textural characteristics of desserts with the addition of sucrose and flower honey, obtained from the curves of the TA.XT plus texture analyzer are shown in Table 4.

The use of flower honey in the formulation of the dessert affects the texture of the finished product and leads to a decrease in the strength of the experimental sample, in comparison with sucrose, which acts as a structuring agent. One of the ways to increase the strength of products using natural sweeteners is to use herbal additives, which are a source of protein components and other nutrients that affect the texture of the product.

In our study, such a nutrient was a developed food supplement from amaranth seeds, which is a source of such structuring components as protein, polysaccharides, polyunsaturated fatty acids, which will not only improve the consumer characteristics of the finished product, but also the nutritional value of products.

Table 4. Textural properties of desserts.

\begin{tabular}{|l|c|c|c|c|}
\hline \multirow{2}{*}{ Samples } & Firmness, $\mathbf{g}$ & Cohesion, g & $\begin{array}{c}\text { Viscosity } \\
\text { index, g.s }\end{array}$ & Viscosity, g.s \\
\cline { 2 - 5 } & & & & \\
\hline $\begin{array}{l}\text { Dessert with } \\
\text { flower honey } \\
\text { without food } \\
\text { additive from } \\
\text { amaranth seeds }\end{array}$ & 158.326 & -39.906 & -541.298 & 3266.576 \\
\hline $\begin{array}{l}\text { Dessert with } \\
\text { flower honey 13 } \\
\% \text { and food } \\
\text { additive from } \\
\text { amaranth seeds } \\
\text { in the amount of } \\
10 \%\end{array}$ & 258.909 & -56.920 & -951.030 & 3850.256 \\
\hline
\end{tabular}

As can be seen from Table 4, the texture properties of dessert using the food additive from amaranth seeds are improved compared to the sample without amaranth seeds. Due to the addition of a food additive from amaranth seeds, the strength of the dessert and the viscosity index increased, which, in technological terms, affects the processes of molding products and the formation of their structure.

Water activity is one of the most important physical characteristic that determines either textural properties of the product or the velocity of chemical and biological processes in it. Data of water activity and moisture content of desserts are presented in Table 5. 
Table 5.Water activity and moisture content of desserts.

\begin{tabular}{|l|c|c|}
\hline \multicolumn{1}{|c|}{ Samples } & Water activity & Moisture content, \% \\
\hline $\begin{array}{l}\text { Dessert with flower honey without food } \\
\text { additive from amaranth seeds }\end{array}$ & 0.947 & 89.1 \\
\hline $\begin{array}{l}\text { Dessert with flower honey 13\% and food } \\
\text { additive from amaranth seeds in the amount } \\
\text { of } 10 \%\end{array}$ & 0.937 & 85.4 \\
\hline
\end{tabular}

As can be seen from Table 3, all samples can be attributed to the group of perishable products, since their water activity index is more than 0.9 .

Thus, in the course of the studies carried out, the optimal concentrations of a food additive from amaranth seeds in an amount of $10 \%$ and flower honey in an amount of $13 \%$ were selected for the production of vanilla cream and the textural characteristics of products were investigated, which showed that the addition of a food additive from amaranth seeds to dessert recipe has a positive effect on the texture of the finished product.

The study showed that dessert with the addition of amaranth seeds and flower honey exceeded the control sample in the content of dietary fiber, vitamins and minerals, the energy value of the test samples decreased, compared to the control sample, the content of proteins, fats and carbohydrates also decreased slightly. Content of B vitamins increased 4.8 times in the recipe of dessert with the addition of amaranth seeds and flower honey compared to the control sample, squalene also appeared in the samples with the addition of amaranth seeds, which is a derivative of vitamin A and with In the synthesis of cholesterol, it turns into its biochemical analogue 7-dehydro-cholesterol, which turns into vitamin D in sunlight, thereby providing radioprotective properties to dessert (protection from radiation). Nutritional and energy value of dessert with amaranth seeds increase due to increased contents of protein, dietary fibre, polyunsaturated fatty acids and other essential nutrients, which makes it possible to recommend such products for overweight persons.

\section{Conclusion}

The feasibility of using food additive from amaranth seeds sort "Polet" was theoretically substantiated and experimentally confirmed in production of dessert. The positive effect of amaranth seeds was found on textural properties and quality indicators of dessert. Additive from amaranth seeds is improved sensory and physicochemical properties of the finished dessert.

This investigation was carried out within of the joint scientific agreement named «Development of formulations hydrogel based desserts with sweeteners» between scientific group from Saratov State Agrarian University, Russia and scientific group from Research Institute of Food Science and Technology, Iran.

\section{References}

1. A.Yu. Popova, V.A. Tutelyan, D.B. Nikityuk, Problems of Nutrition, 90, 4 (2021)

2. V.A. Tutelyan, D.B. Nikityuk, A.K. Baturin, A.V. Vasiliev, M.M.G. Gapparov, N.V. Zhilinskaya, et al., Problems of Nutrition, 89, 4 (2020)

3. C.W. Walter, Free Press New York (2001)

4. T.V. Chirkova, Sorovsk educational journal, 10 (1999)

5. I.M. Zharkova, L.A. Miroshnichenko, A.A. Zvyagina, I.A. Bavykina, Problems of Nutrition, 83, 1 (2014) 
6. Yu. F. Roslyakov, N. A. Shmalko, L. K. Bochkova, Food Technology, 4 (2004)

7. H.-P. He, Y. Cai, M. Sun, H. Corke, J. Agric. Food Chem., 50 (2002)

8. Y. Tang, R. Tsao, Molecular Nutrition \& Food Research, 61, 7 (2017)

9. S. D'Amico, R. Schoenlechner, Gluten-free ancient grains (2017)

10. V.P. Borovikov Statistica. Art of data analysis on a computer for professionals (SaintPetersburg, Russian. Peter., 2003) 